Cardiopulmonary bypass was established by bicaval cannulation and common femoral arterial return. A parallel 0.25 inch cannula was connected between the arterial return and the SVC cannula by means of Y connectors to provide RCP after HCA. This was primed and clamped at both ends. After implementation of $\mathrm{HCA}$ (nasopharyngeal temperature $15^{\circ} \mathrm{C}$ ), the femoral arterial cannula was clamped and the blood was drained into the cardiotomy reservoir. The SVC cannula was isolated from the venous circulation, and RCP commenced with flow (mean $383 \pm 35 \mathrm{ml} / \mathrm{min}$ ) adjusted to maintain a right jugular bulb pressure of $25 \mathrm{~mm} \mathrm{Hg}$ or less. A dedicated retrograde right internal jugular line independent of any drug infusions was used to measure jugular bulb pressure. A detailed description of our RCP circuit can be found elsewhere. ${ }^{3}$

Before circulatory arrest, a portable gamma camera (Siemens LEM+; Siemens AG, Erlangen, Germany) linked to a dedicated nuclear medicine computer system (Gamma 11, Nuclear Diagnostics, Kent, United Kingdom) was placed anterior to the head of the patient. After HCA and immediately before the commencement of RCP, $100 \mathrm{MBq}$ of ${ }^{99 \mathrm{~m}} \mathrm{Tc}-\mathrm{HMPAO}$ was administered into the cardiotomy reservoir and allowed to diffuse. Planar dynamic brain imaging was commenced when the first sign of activity was seen in the SVC. Images were acquired every 2 seconds for the first 2 minutes and every minute thereafter for the duration of RCP (mean $32 \pm 7$ minutes). Inspection of the gamma camera images showed ${ }^{99 \mathrm{~m}}$ Tc-HMPAO activity spreading quickly from the right jugular bulb through the cerebral vascular tree (Fig. 1) and throughout the brain within 3 minutes (Fig. 2). Continuous ${ }^{99 \mathrm{~m}} \mathrm{Tc}-\mathrm{HMPAO}$ accumulation was seen in the gray and white matter for the duration of RCP, with reduction of activity in the superior sagittal sinus as the agent was cleared from the blood pool. Time activity curves constructed for both cerebral hemispheres and a background region in the neck demonstrate homogeneous perfusion in both cerebral hemispheres (Fig. 3).

One of the patients, who had previously undergone a repeated aortic valve replacement and coronary artery revascularization and had dissecting type $\mathrm{A}$ aortic aneurysm and vein graft occlusion, died of myocardial failure in the early postoperative period. Assessment of neurologic function was not possible because the patient was electively sedated during the postoperative course. The remaining two patients had normal return to consciousness in less then 24 hours after operation and had no neurologic complications.

This pilot study demonstrates that cerebral perfusion does occur with RCP in human beings. The question of whether the metabolic demands of the brain may be met with this technique remains to be answered.

\section{REFERENCES}

1. Usui A, Hotta T, Hiroura M, et al. Retrograde cerebral perfusion through a superior vena caval cannula protects the brain. Ann Thorac Surg 1992;53:47-53.

2. Costa DC, Ell PJ, Cullum ID, Jorritt PH. The in vivo distribution of ${ }^{99} \mathrm{Te}^{\mathrm{m}}-\mathrm{HM}-\mathrm{PAO}$ in normal man. Nucl Med Commun 1986;7:647-58.

3. Pagano D, Carey JA, Patel RL, et al. Retrograde cerebral perfusion: clinical experience in emergency and elective aortic operations. Ann Thorac Surg 1995;59:393-7.

\title{
RETRANSFUSION OF THORACIC WOUND BLOOD DURING HEART SURGERY OBSCURES BIOCOMPATIBILITY OF THE EXTRACORPOREAL CIRCUIT
}

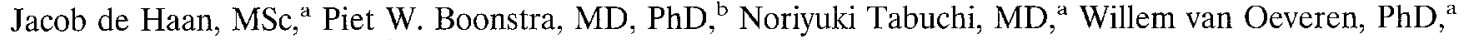
and Tjark Ebels, $\mathrm{MD}, \mathrm{PhD},{ }^{\mathrm{b}}$ Groningen, The Netherlands

To minimize blood trauma and postoperative pathologic outcomes, it is of great concern to improve the biocompatibility of extracorporeal circuits.' Worldwide, many comparative studies have been performed to select the most biocompatible extracorporeal circuit. In many of these studies, however, a systematic error was made.

From the Blood Interaction Research Division ${ }^{a}$ and the Department of Cardiothoracic Surgery, University Hospital Groningen, The Netherlands.

Accepted for publication May 31, 1995.

J Thorac Cardiovasc Surg 1996;111:272-5

Copyright $(\mathcal{C} 1996$ by Mosby-Year Book, Inc.

$0022-5223 / 96 \$ 5.00+0 \quad \mathbf{1 2 / 5 4 / 6 6 6 0 4}$
Retransfusion of heparinized blood from the wound area is an established method to attenuate excessive blood loss during cardiopulmonary bypass (CPB). Retransfusion of this wound blood is feasible by suction from the thoracic cavity and recirculation through the cardiotomy reservoir. Because wound blood is highly traumatized, as we demonstrated in recent studies, ${ }^{2,3}$ there are likely to be significant effects of wound blood retransfusion on the systemic blood, depending on the amount of retransfused wound blood. Particularly, retransfusion of the damaged wound blood could obscure possible improvements in biocompatibility of extracorporeal circuits. ${ }^{4}$ In this study, wound blood was retained during CPB in one group of 14 patients and retransfused only at the end of bypass. The control group consisted of 20 other patients, who were 


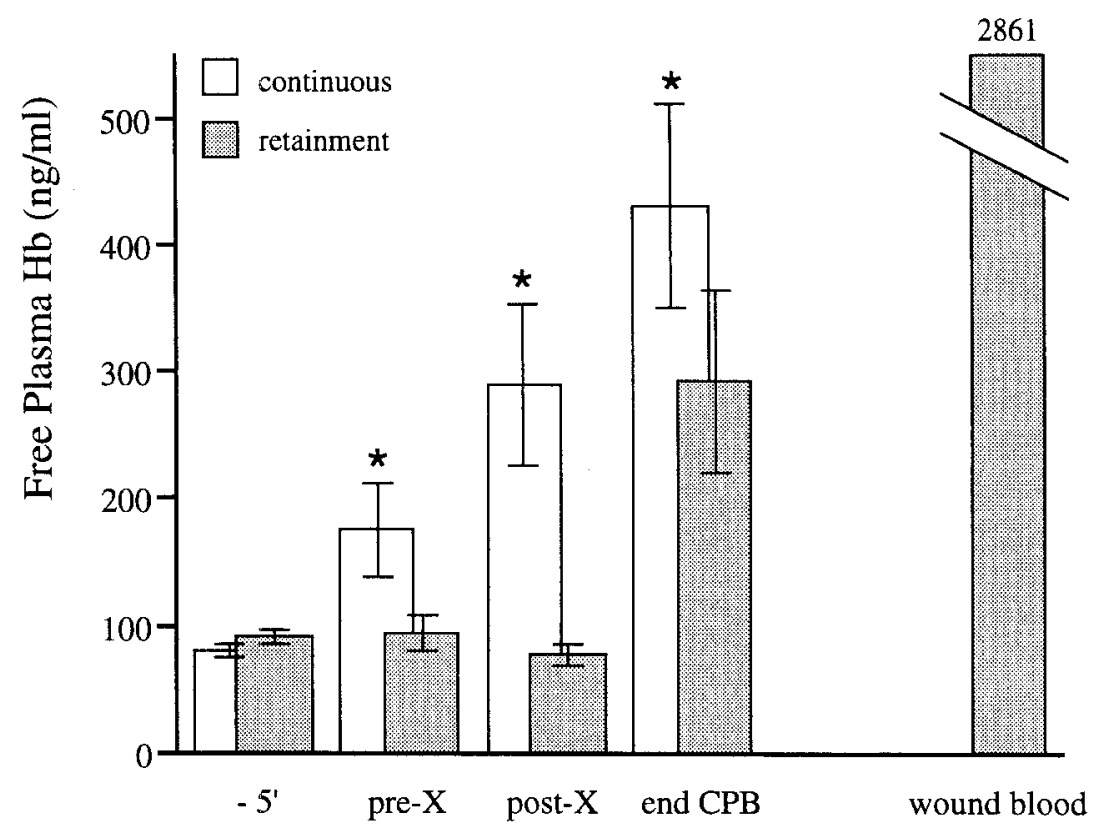

Fig. 1. The degree of hemolysis during CPB is reflected by plasma levels of hemoglobin (Free Plasma Hb). Mean values of the patient group with continuous retransfusion of wound blood are indicated by the white bars; mean values of the patient group with retainment of wound blood are indicated by gray bars. The sample points indicate 5 minutes after heparinization and before start of CPB $\left(-5^{\prime}\right)$, before release of the aortic crossclamp (pre- $X$ ), after release of the aortic crossclamp (post-X), and at the end of CPB (end CPB). The wound blood bar indicates the concentration of free hemoglobin in the wound blood during retention. Error bars indicate the standard error of the mean. An asterisk indicates a significant difference $(p<0.01$ by unpaired Student's $t$ test) between both groups at a specific sample point.

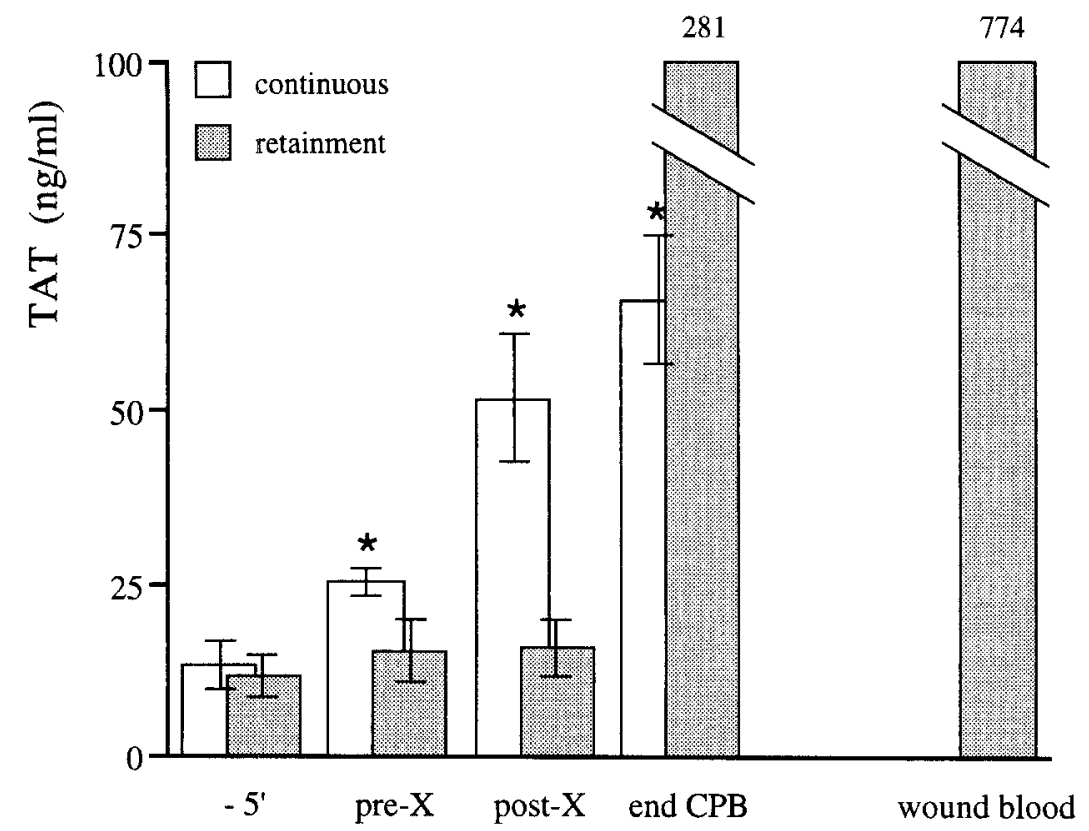

Fig. 2. The degree of clotting activation during CPB is reflected by plasma levels of TAT III (TAT). All other graph specifications are the same as those in Fig. 1. 


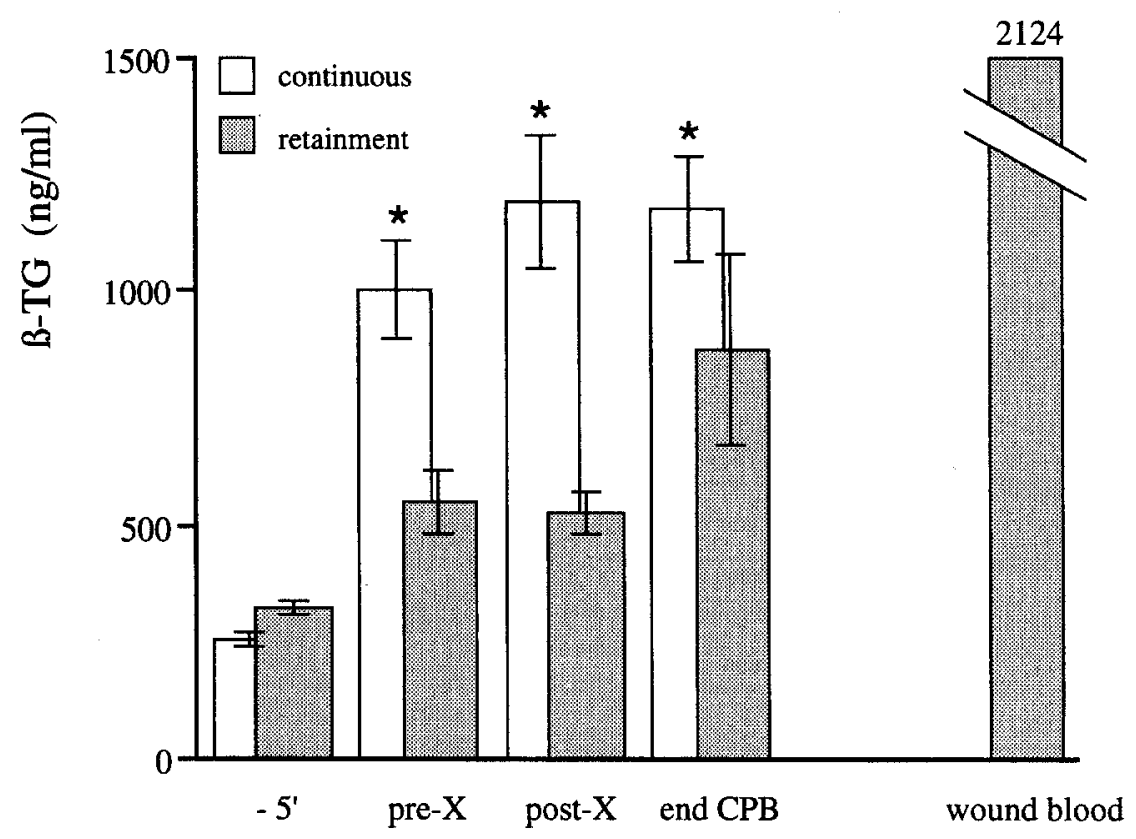

Fig. 3. The degree of platelet degranulation during CPB is reflected by plasma levels of $\beta$-TG. All other graph specifications are the same as those in Fig. 1.

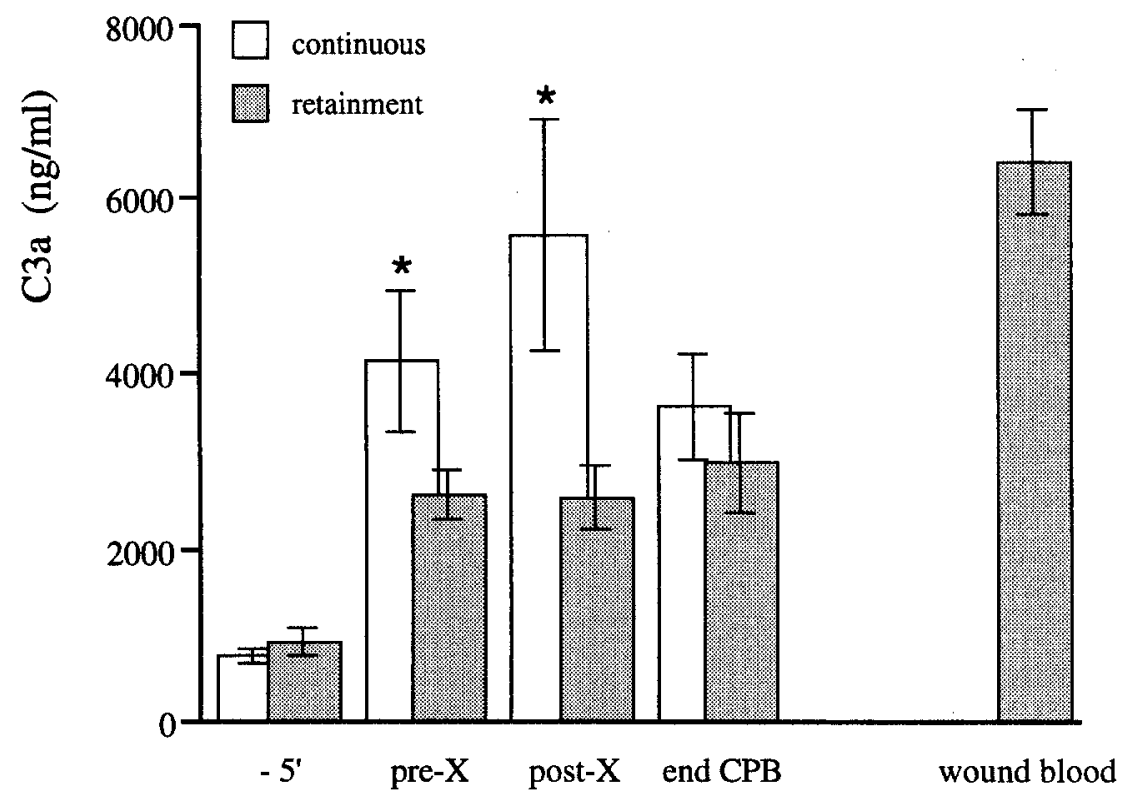

Fig. 4. The degree of complement activation during CPB is reflected by plasma levels of complement factor $\mathrm{C} \mathrm{a}_{\text {desArg }}(C 3 a)$. All other graph specifications are the same as those in Fig. 1.

subjected to immediate and continuous retransfusion of wound blood during the operation. Blood damage and activation during operation were assessed by measuring the plasma levels of variables indicative of hemolysis (Fig. 1), clotting (Fig. 2), platelet degranulation (Fig. 3), and complement activation (Fig. 4). The validity of these variables in reflecting extracorporeal related blood damage could thus be evaluated.

None of the patients subjected to elective CPB received any drugs known to affect the clotting system or platelets during the last 7 days before the CPB bypass procedure. All circuits were primed with $2000 \mathrm{ml}$ of oxypolygelatin 
(Gelifundol; Biotest Pharma GmbH, Dreiech, Germany) and 1500 IU of bovine heparin (Leo, Emmen, The Netherlands). Heparinization was achieved by introduction of $300 \mathrm{IU} / \mathrm{kg}$ heparin intravenously, with additional heparin if the activated clotting time fell under 400 seconds. Samples for biochemical assays were taken 5 minutes after heparinization (and before start of CPB), just before release of the aortic crossclamp, immediately after release of the aortic crossclamp, and at the end of bypass (after the retransfusion of the wound blood in the retainment group). In 14 patients, the wound blood was sucked into a separate container connected to the cardiotomy reservoir and retained during the surgical procedure. Before retransfusion of this blood at the end of $\mathrm{CPB}$, the retained wound blood was also sampled. Hemolysis was determined by measuring levels of free plasma hemoglobin by a spectrophotometric technique ${ }^{5}$ (Fig. 1). Clotting activation was reflected by plasma levels of thrombinantithrombin III complex (TAT III, Fig. 2), determined by enzyme-linked immunosorbent assay (Behring, Marburg, Germany); platelet degranulation was reflected by plasma levels $\beta$-thromboglobulin ( $\beta$-TG; Fig. 3 ), determined by radioimmunoassay (Kodak, Amersham, U.K.). Complement activation was reflected by plasma levels $\mathrm{C} 3 \mathrm{a}_{\text {desArg }}$ (Fig. 4), determined by enzyme-linked immunosorbent assay (Quidel, San Diego, Calif.). Because of the hemodilution by admixture of prime solution from the extracorporeal circuit, all obtained data were corrected for hematocrit before further evaluation.

The effect of retransfusing wound blood on circulating concentrations of products such as free hemoglobin and TAT III was demonstrated by the immediate increase in the blood retention group after the retransfusion and the more gradual and lesser increase in patients with continuous retransfusion. Furthermore, the lack of any noticeable increase of these variables before retransfusion endorses the dominating effect of wound blood retransfusion on free hemoglobin and TAT III levels. Moreover, the high concentrations of free hemoglobin and TAT III in the wound blood explain the observed increases satisfactorily.

Concentrations of $\beta$-TG also increased, in particular after the retransfusion of wound blood. A significant increase of $\beta$-TG in the retainment group was also observed, however, during the first phase of CPB before release of the aortic crossclamp and before any retransfusion of wound blood. This increase, independent of retransfusion, strongly suggests that the extracorporeal circuit contributes significantly to platelet damage. Finally, the complement system was activated in both groups of patients, as indicated by the increasing C3a concentrations after the start of CPB, although to a much lesser extent in the blood retention group than in patients who underwent continuous retransfusion. The common $\mathrm{C} 3 \mathrm{a}$ generation indicates complement activation by the extracorporeal circuit in all patients. ${ }^{6}$ The differences between groups is explainable by the method of wound blood retransfusion. It is likely that blood complement in the wound is activated through the classic pathway, resulting in $\mathrm{C} 3 \mathrm{a}$ levels similar to the levels in circulation. The increase in systemic $\mathrm{C} 3 \mathrm{a}$ level after continuous retransfusion or re- transfusion of retained wound blood suggests that infusion of denatured or substances unfamiliar to blood is triggering an additional systemic complement activation response. ${ }^{7}$

In conclusion, our study demonstrates that data obtained in previous clinical studies that focused on biocompatibility assessment of extracorporeal circuits were biased when substantial amounts of wound blood were retransfused during the procedure. Blood activation related to clotting, platelet degranulation, complement activation, and hemolysis by the extracorporeal circuit during $\mathrm{CPB}$-as indicated by TAT III, $\beta$-TG, C3a, and free hemoglobin-is vastly influenced by the retransfusion of wound blood. Moreover, free hemoglobin and TAT III generation related to the extracorporeal circuit were found insignificant. Variables such as TAT III and hemolysis are therefore unfit to analyze and quantify the biocompatibility of extracorporeal circuits. Furthermore, biocompatibility of extracorporeal circuits, as analyzed by variables such as $\mathrm{C} 3 \mathrm{a}$ and $\beta$-TG, cannot be evaluated with the presently used technique for heart operations of continuously retransfusing wound blood. We suggest that the wound blood be retained during assessment of the biocompatibility of extracorporeal circuits or that the wound blood be washed by cell-saving techniques, to wash out the disturbing plasma factors before retransfusion.

\section{REFERENCES}

1. Van Oeveren W, Wildevuur CR, Kazatchkine MD. Biocompatibility of extracorporeal circuits in heart surgery. Transfus Sci 1990;11:5-33.

2. Tabuchi N, de Haan J, Boonstra PW, van Oeveren W. Activation of fibrinolysis in the pericardial cavity during cardiopulmonary bypass. J Thorac Cardiovasc SURG 1993;106:828-33.

3. Boonstra PW, van Imhoff GW, Eysman L. Reduced platelet activation and improved hemostasis after controlled cardiotomy suction during clinical membrane oxygenator perfusions. J Thorac Cardiovasc Surg 1985;89:900-6.

4. Sundaram S, Irvine L, Courtney JM, Taggart DP, Wheatley DJ, Lowe GD. Patterns of blood response during cardiopulmonary bypass. Int $\mathbf{J}$ Artif Organs 1992;15:243-8.

5. Harboe H. A method for determination of haemoglobin in plasma by near UV spectroscopy. Scand Clin Lab Invest 1959;11:66-70.

6. Jones HM, Matthews N, Vaughan RS, Stark JM. Cardiopulmonary bypass and complement activation: involvement of classical and alternative pathways. Anaesthesia 1982;37:629-33.

7. Videm V, Fosse E, Mollnes TE, Garred P, Svennevig JL. Time for new concepts about measurement of complement activation by cardiopulmonary bypass? Ann Thorac Surg 1992;54:725-31. 